\title{
Vector-type four-quark interaction and its impact on QCD phase structure
}

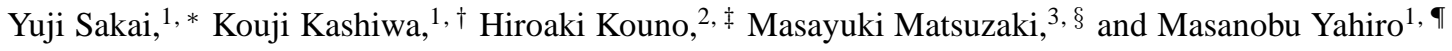 \\ ${ }^{1}$ Department of Physics, Graduate School of Sciences, Kyushu University, Fukuoka 812-8581, Japan \\ ${ }^{2}$ Department of Physics, Saga University, Saga 840-8502, Japan \\ ${ }^{3}$ Department of Physics, Fukuoka University of Education, Munakata, Fukuoka 811-4192, Japan
}

(Dated: June 20, 2018)

\begin{abstract}
Effects of the vector-type four-quark interaction on QCD phase structure are investigated in the imaginary chemical potential $(\mu)$ region, by using the Polyakov-loop extended Nambu-Jona-Lasinio (PNJL) model with the extended $\mathbb{Z}_{3}$ symmetry. In the course to this end, we clarify analytically the Roberge-Weiss periodicity and symmetry properties of various quantities under the existence of a vector-type four-quark interaction. In the imaginary $\mu$ region, the chiral condensate and the quark number density are sensitive to the strength of the interaction. Based on this result, we propose a possibility to determine the strength of the vector-type interaction, which largely affects QCD phase structure in the real $\mu$ region, by comparing the results of lattice simulations and effective model calculations in the imaginary $\mu$ region.
\end{abstract}

PACS numbers: 11.30.Rd, 12.40.-y

\section{INTRODUCTION}

The progress in computer power has made it feasible to do realistic simulations in the lattice QCD for finite temperature $(T)$ system without quark chemical potential $(\mu)$ [1]. As for $\mu^{2}>0$, however, lattice QCD has the well-known sign problem, and then the results are still far from perfection; for example, see Ref. [2] and references therein. Several approaches have been proposed to solve the sign problem. One of them is the use of imaginary chemical potential, since the fermionic determinant that appears in the Euclidean partition function is real in the case; for example, see Refs. [3, 4, 5] and references therein.

When physical quantities are available with lattice QCD in the imaginary $\mu$ region, in principle it is possible to extrapolate them to real $\mu$, until there appears a discontinuity. Actually, such an extrapolation was made for the phase transition curve by assuming some analytic functions for the curve [3, 4]. This direct extrapolation may work for small real $\mu / T$, but its accuracy is quite unknown for large real $\mu / T$ [6]. This problem may be circumvented by the effective theory that can evaluate the partition function in both the real and imaginary $\mu$ regions and reproduce the results of lattice QCD in the imaginary $\mu$ region, if such an effective theory is found.

In the region of imaginary chemical potential $\mu=i T \theta$, Roberge and Weiss (RW) found [7] that the partition function $Z(\theta)$ of $\mathrm{SU}(N)$ gauge theory is a periodic function of $\theta$ with a period $2 \pi / N$, showing that $Z(\theta+2 \pi k / N)$ is reduced to $Z(\theta)$ with the $\mathbb{Z}_{N}$ transformation for any integer $k$. The RW periodicity means that $Z(\theta)$ is invariant under the combination of the $\mathbb{Z}_{N}$ transformation and another transformation $\theta \rightarrow \theta+$

\footnotetext{
*sakai@phys.kyushu-u.ac.jp

†kashiwa@phys.kyushu-u.ac.jp

抽ounoh@cc.saga-u.ac.jp

$\S$ matsuza@fukuoka-edu.ac.jp

"yahiro@phys.kyushu-u.ac.jp
}

$2 \pi k / N$, that is, under the extended $\mathbb{Z}_{N}$ transformation [6]

$$
q \rightarrow U q, A_{\nu} \rightarrow U A_{\nu} U^{-1}-\frac{i}{g}\left(\partial_{\nu} U\right) U^{-1}, \theta \rightarrow \theta+\frac{2 \pi k}{N},(1)
$$

where $U(x, \tau)$ are elements of $\mathrm{SU}(N)$ with the boundary condition $U(x, \beta)=\exp (-2 i \pi k / N) U(x, 0), q$ is the fermion field, $A_{\nu}$ is the gauge field and $\beta$ is the inverse of temperature $T$. Quantities invariant under the extended $\mathbb{Z}_{N}$ transformation, such as the thermodynamic potential $\Omega(\theta)$ and the chiral condensate, keep the RW periodicity. Meanwhile, the Polyakov loop $\Phi$ is not invariant under the transformation (1), since it is transformed as $\Phi \rightarrow \Phi \exp (-i 2 \pi k / N)$. Such a non-invariant quantity does not have the periodicity, but this problem can be solved by the modified Polyakov loop defined later in Eq. (12) invariant under the extended $\mathbb{Z}_{N}$ transformation [6]. Roberge and Weiss also showed with perturbation that in the high $T$ region $d \Omega(\theta) / d \theta$ and $\Phi(\theta)$ are discontinuous at $\theta=(2 k+1) \pi / N$, and also found with the strongly coupled lattice theory that the discontinuities disappear in the low $T$ region. The first-order RW phase transition of $d \Omega(\theta) / d \theta$, that is, the quark number density $n=$ $-d \Omega / d \mu=i(d \Omega(\theta) / d \theta) / T$ was observed in lattice simulations [3, 4, 5].

The Nambu-Jona-Lasinio (NJL) model [8] is famous as a model for understanding chiral symmetry breaking, but it does not have the extended $\mathbb{Z}_{3}$ symmetry as well as the confinement mechanism. In the previous work [6], we pointed out that the Polyakov-loop extended NJL (PNJL) model designed to have the confinement mechanism [9, 10, 11, 12, $13,14,15,16,17,18,19,20,21,22,23,24,25,26]$ possesses the extended $\mathbb{Z}_{3}$ symmetry. Hence the thermodynamic potential $\Omega(\theta)$ of the PNJL model has the RW periodicity. Using the RW periodicity and the $\theta$-evenness of $\Omega(\theta)$, $\Omega(\theta)=\Omega(-\theta)$, we also found that $\theta$-odd $(\theta$-even) quantities such as $n(\sigma)$ have first-order (second-order) phase transitions on lines $\theta=(2 k+1) \pi / 3$ in the $\theta-T$ plane. This result is consistent with the results of lattice QCD and even more informative. Thus, the extended $\mathbb{Z}_{3}$ symmetry is essential. The extended $\mathbb{Z}_{3}$ symmetry of finite $\theta \mathrm{QCD}$ is an extension of the $\mathbb{Z}_{3}$ symmetry of pure gauge QCD. 
The confinement mechanism is a key to understand the physical real $\mu$ world. Actually, it is predicted that the mechanism largely shifts the critical endpoint [27] toward higher $T$ and lower $\mu$ than the NJL model predicts [17, 22, 24]. In contrast, it is known [22, 24, 28, 29] that the vector-type fourquark interaction $\left(\bar{q} \gamma_{\mu} q\right)^{2}$ largely moves the critical endpoint in the opposite direction, if it is newly added to the NJL and PNJL models. Thus, it is essential from the phenomenological point of view to determine the strength of the vector-type four-quark interaction.

So far, the vector-type interaction was often ignored in the NJL and PNJL models. In the relativistic meson-nucleon theory [30], meanwhile, the repulsive force mediated by vector mesons is essential to account for the saturation property of nuclear matter. Using the auxiliary field method, one can convert quark-quark interactions to meson-quark interactions; for example, see Refs. [31, 32, 33] and references therein. In the hadron phase, furthermore, quarks have a large effective mass as a result of spontaneous chiral symmetry breaking, and then nucleons can be considered to be formed from such three heavy quarks, that is, three constituent quarks. It is then natural to think that there exists a correspondence between the meson-nucleon interactions and the quark-quark interactions. In this point of view, it is quite likely that the vector-type fourquark interaction is not negligible and even significant in particular at the finite quark-density region corresponding to the nuclear saturation density.

In this paper we investigate roles of the vector-type fourquark interaction in the imaginary $\mu$ region and show that the chiral condensate $\sigma$ and the quark number density $n$ are quantities sensitive to the strength of vector-type four-quark interaction. We then propose to measure $n$ as well as $\sigma$ with lattice QCD. If the strength of vector-type four-quark interaction is determined from the measured $\sigma$ and $n$, the PNJL model with the interaction can predict the phase diagram in the real $\mu$ region with reasonable reliability. The previous proof [6] on the RW periodicity and the even/odd property of the extended $\mathbb{Z}_{3}$ invariant quantities is not applicable to the case with vectortype four-quark interaction. We then prove the properties in a way different from the previous one [6].

In sec. II we present the PNJL model with the vector-type four-quark interaction, and derive the thermodynamic potential invariant under the extended $\mathbb{Z}_{3}$ transformation. For the case of imaginary $\mu=i T \theta$, it is proven that the extended $\mathbb{Z}_{3}$ invariant quantities exhibit the RW periodicity. For both of imaginary and real $\mu$ cases, their even/odd properties are determined. It is shown from the the RW periodicity and the even/odd properties that the RW phase transition appearing in $\theta=(2 k+1) \pi / 3$ is a family of first-order phase transitions in $\theta$-odd quantities and second-order ones in $\theta$-even quantities. In sec. III we show results of numerical calculations. Section IV is devoted to Summary.

\section{PNJL MODEL}

\section{A. Formulation}

We consider the two-flavor PNJL model with the vectortype four-quark interaction. The PNJL Lagrangian is

$$
\begin{gathered}
\mathcal{L}=\bar{q}\left(i \gamma_{\nu} D^{\nu}-m_{0}\right) q+G_{\mathrm{s}}\left[(\bar{q} q)^{2}+\left(\bar{q} i \gamma_{5} \vec{\tau} q\right)^{2}\right] \\
-G_{\mathrm{v}}\left(\bar{q} \gamma_{\mu} q\right)^{2}-\mathcal{U}\left(\Phi[A], \Phi_{\mathrm{H}}[A], T\right)
\end{gathered}
$$

where $q$ denotes the quark field, $m_{0}$ does the current quark mass, $\vec{\tau}$ stands for the isospin matrix, and $D^{\nu}=\partial^{\nu}-i A^{\nu}-$ $\delta_{0}^{\nu} \mu$ for the complex chemical potential $\mu=\mu_{\mathrm{R}}+i T \theta$. Parameters $G_{\mathrm{S}}$ and $G_{\mathrm{v}}$ represent the coupling constants of the scalarand vector-type four-quark interactions, $(\bar{q} q)^{2}$ and $\left(\bar{q} \gamma_{\mu} q\right)^{2}$, respectively. The Polyakov potential $\mathcal{U}$, defined later in (9), is a function of the Polyakov loop $\Phi$ and its Hermitian conjugate $\Phi_{\mathrm{H}}$,

$$
\Phi=\frac{1}{N} \operatorname{Tr} L, \quad \Phi_{\mathrm{H}}=\frac{1}{N} \operatorname{Tr} L^{\dagger}
$$

with

$$
L(\mathbf{x})=\mathcal{P} \exp \left[i \int_{0}^{\beta} d \tau A_{4}(\mathbf{x}, \tau)\right]
$$

where $A_{4}=i A_{0}, N=3$, and $\mathcal{P}$ is the path ordering. In the PNJL model, $\Phi$ and $\Phi_{\mathrm{H}}$ are treated as classical variables. The temporal component $A_{4}$ is diagonal in the flavor space, because the color and the flavor space are completely separated out in the present case. In the Polyakov gauge, $L$ can be written in a diagonal form in the color space [11]:

$$
L=e^{i \beta\left(\phi_{3} \lambda_{3}+\phi_{8} \lambda_{8}\right)}=\operatorname{diag}\left(e^{i \beta \phi_{a}}, e^{i \beta \phi_{b}}, e^{i \beta \phi_{c}}\right),
$$

where $\phi_{a}=\phi_{3}+\phi_{8} / \sqrt{3}, \phi_{b}=-\phi_{3}+\phi_{8} / \sqrt{3}$ and $\phi_{c}=$ $-\left(\phi_{a}+\phi_{b}\right)=-2 \phi_{8} / \sqrt{3}$.

The Polyakov loop $\Phi$ is an exact order parameter of the spontaneous $\mathbb{Z}_{3}$ symmetry breaking in the pure gauge theory. Although the $\mathbb{Z}_{3}$ symmetry is not exact in the system with dynamical quarks, it still seems to be a good indicator of the deconfinement phase transition. Therefore, we use $\Phi$ to define the deconfinement phase transition.

Making the mean field approximation (MFA), one can get the Lagrangian density

$$
\begin{array}{r}
\mathcal{L}_{\mathrm{MFA}}=\bar{q}\left(i \gamma_{\mu} D^{\mu}+\gamma_{0} \Sigma_{\mathrm{v}}-\left(m_{0}+\Sigma_{\mathrm{s}}\right)\right) q \\
-U(\sigma, n)-\mathcal{U}\left(\Phi, \Phi_{\mathrm{H}}, T\right)
\end{array}
$$

with

$$
\begin{aligned}
\sigma & =\langle\bar{q} q\rangle, \quad n=\left\langle\bar{q} \gamma_{0} q\right\rangle, \quad \Sigma_{\mathrm{s}}=-2 G_{\mathrm{s}} \sigma \\
\Sigma_{\mathrm{v}} & =-2 G_{\mathrm{v}} n, \quad U=G_{\mathrm{s}} \sigma^{2}-G_{\mathrm{v}} n^{2}
\end{aligned}
$$

and obtain the thermodynamic potential $\Omega$ by making the path 
integral over the quark field:

$$
\begin{aligned}
\Omega & =-2 N_{f} \int \frac{d^{3} \mathrm{p}}{(2 \pi)^{3}}[3 E(\mathrm{p}) \\
& +\frac{1}{\beta} \ln \left[1+3\left(\Phi+\Phi_{\mathrm{H}} e^{-\beta E^{-}(\mathbf{p})}\right) e^{-\beta E^{-}(\mathbf{p})}+e^{-3 \beta E^{-}(\mathbf{p})}\right] \\
& \left.+\frac{1}{\beta} \ln \left[1+3\left(\Phi_{\mathrm{H}}+\Phi e^{-\beta E^{+}(\mathbf{p})}\right) e^{-\beta E^{+}(\mathbf{p})}+e^{-3 \beta E^{+}(\mathbf{p})}\right]\right] \\
& +U+\mathcal{U},
\end{aligned}
$$

with $E(\mathrm{p})=\sqrt{\mathbf{p}^{2}+M^{2}}, M=m_{0}+\Sigma_{\mathrm{s}}$ and $E^{ \pm}(\mathrm{p})=$ $E(\mathrm{p}) \pm \tilde{\mu}$. Here the effective chemical potential $\tilde{\mu}$ is defined by $\tilde{\mu}=\mu_{\mathrm{R}}+i T \theta-2 G_{\mathrm{v}} n$.

We use $\mathcal{U}$ of Ref. [14]:

$$
\begin{aligned}
& \frac{\mathcal{U}}{T^{4}}=-\frac{b_{2}(T)}{2} \Phi_{\mathrm{H}} \Phi-\frac{b_{3}}{6}\left(\Phi_{\mathrm{H}}{ }^{3}+\Phi^{3}\right)+\frac{b_{4}}{4}\left(\Phi_{\mathrm{H}} \Phi\right)^{2}, \\
& b_{2}(T)=a_{0}+a_{1}\left(\frac{T_{0}}{T}\right)+a_{2}\left(\frac{T_{0}}{T}\right)^{2}+a_{3}\left(\frac{T_{0}}{T}\right)^{3} .
\end{aligned}
$$

Parameters of $\mathcal{U}$ are fitted to results of lattice simulations in the pure gauge system with finite $T$ [34, 35]. In the pure gauge system the parameter $T_{0}$ agrees with the critical temperature
$T_{\mathrm{D}}$ of deconfinement phase transition. Hence, as a reasonable choice, one can adjust $T_{0}$ to $T_{\mathrm{D}}=270 \mathrm{MeV}$ of pure gauge lattice simulations. However, the PNJL model with this value of $T_{0}$ yields somewhat larger value of $T_{\mathrm{D}}$ than $\sim 173 \mathrm{MeV}$ predicted by the full LQCD simulation [36, 37, 38]. In this paper, we then take the rescaled value $T_{0}=190 \mathrm{MeV}$ that gives $T_{\mathrm{D}}=176 \mathrm{MeV}$ in the PNJL calculation with $\mu=0$ [6].

As expected, $\Omega$ is invariant under the extended $\mathbb{Z}_{3}$ transformation,

$$
\begin{aligned}
& e^{ \pm i \theta} \rightarrow e^{ \pm i \theta} e^{ \pm i \frac{2 \pi k}{3}}, \quad \Phi(\theta) \rightarrow \Phi(\theta) e^{-i \frac{2 \pi k}{3}}, \\
& \Phi_{\mathrm{H}}(\theta) \rightarrow \Phi_{\mathrm{H}}(\theta) e^{i \frac{2 \pi k}{3}} .
\end{aligned}
$$

Thus, the extended $\mathbb{Z}_{3}$ invariance of $\Omega$ is held, even if the vector-type four-quark interaction is added to the PNJL Langrangian. In general, the symmetry persists, even if any sort of multi-quark interactions are added to the NJL sector.

As mentioned above, it is essential to introduce the modified Polyakov loop,

$$
\Psi \equiv e^{i \theta} \Phi, \quad \Psi_{\mathrm{H}} \equiv e^{-i \theta} \Phi_{\mathrm{H}},
$$

invariant under the the extended $\mathbb{Z}_{3}$ transformation (11).

The extended $\mathbb{Z}_{3}$ transformation is then rewritten into

$$
e^{ \pm i \theta} \rightarrow e^{ \pm i \theta} e^{ \pm i \frac{2 \pi k}{3}}, \quad \Psi(\theta) \rightarrow \Psi(\theta), \quad \Psi_{\mathrm{H}}(\theta) \rightarrow \Psi_{\mathrm{H}}(\theta),
$$

and $\Omega$ is also into

$$
\begin{aligned}
\Omega=-2 N_{f} \int \frac{d^{3} \mathrm{p}}{(2 \pi)^{3}}[3 E(\mathrm{p}) & +\frac{1}{\beta} \ln \left[1+3 \Psi e^{-\beta E(\mathbf{p})} e^{\beta \hat{\mu}_{n}}+3 \Psi_{\mathrm{H}} e^{-2 \beta E(\mathbf{p})} e^{2 \beta \hat{\mu}_{n}} e^{3 i \theta}+e^{-3 \beta E(\mathbf{p})} e^{3 \beta \hat{\mu}_{n}} e^{3 i \theta}\right] \\
+ & \left.\frac{1}{\beta} \ln \left[1+3 \Psi_{\mathrm{H}} e^{-\beta E(\mathbf{p})} e^{-\beta \hat{\mu}_{n}}+3 \Psi e^{-2 \beta E(\mathbf{p})} e^{-2 \beta \hat{\mu}_{n}} e^{-3 i \theta}+e^{-3 \beta E(\mathbf{p})} e^{-3 \beta \hat{\mu}_{n}} e^{-3 i \theta}\right]\right]+U \\
& -\frac{b_{2}(T) T^{4}}{2} \Psi_{\mathrm{H}} \Psi-\frac{b_{3} T^{4}}{6}\left(\Psi_{\mathrm{H}}^{3} e^{3 i \theta}+\Psi^{3} e^{-3 i \theta}\right)+\frac{b_{4} T^{4}}{4}\left(\Psi_{\mathrm{H}} \Psi\right)^{2}
\end{aligned}
$$

where $\hat{\mu}_{n}=\mu_{\mathrm{R}}-2 G_{\mathrm{v}} n$, and the factor $\exp ( \pm i 3 \theta)$ is also invariant under the transformation (13). Variables $X=$ $\Psi, \Psi_{\mathrm{H}}, \sigma$ and $n$ are determined from the stationary conditions

$$
\partial \Omega / \partial X=0,
$$

by solving the equations for $X$. The thermodynamic potential $\Omega(\theta)$ at each $\theta$ is obtained by inserting the solutions into (14). Such a calculation is possible for any complex $\mu$.

\section{B. RW periodicity and even/odd property}

We begin with the case of imaginary chemical potential $\mu=i T \theta$, that is, take $\mu_{\mathrm{R}}=0$ in (14). The equations (15) depend on $\theta$ only through the factor $\exp (3 i \theta)$, so that the solutions $X$ are functions of the factor:

$$
X=X\left(e^{3 i \theta}\right) .
$$

Since $\Omega(\theta)$ is obtained by inserting the solutions $X$ into $[8]$, $\Omega(\theta)$ is also a function of $\exp (3 i \theta)$. Hence, we get

$$
\Omega\left(\theta+\frac{2 \pi k}{3}\right)=\Omega(\theta), \quad X\left(\theta+\frac{2 \pi k}{3}\right)=X(\theta) .
$$

Thus, quantities invariant with respect to the extended $\mathbb{Z}_{3}$ transformation have the RW periodicity. It is found from the proof shown above that the property is not influenced by the presence/absence of the four-quark vector-type interaction. This will be confirmed later through Fig 1 .

Next we discuss their more detailed symmetry properties. Taking the complex conjugate to (14), one can find that the complex conjugate $\Omega^{*}$ has the same form as the original one $\Omega$, if $\sigma^{*}, n^{*}, \Psi^{*}$ and $\Psi_{\mathrm{H}}^{*}$ are replaced by $\sigma$ and $-n, \Psi_{\mathrm{H}}$ and $\Psi$, respectively. This indicates that the solutions $X^{*}$ of $\partial \Omega^{*} / \partial X^{*}=0$ are related to those $X$ of $\partial \Omega / \partial X=0$ as

$$
\sigma^{*}=\sigma, n^{*}=-n, \Psi^{*}=\Psi_{\mathrm{H}}, \Psi_{\mathrm{H}}^{*}=\Psi .
$$


The last two equations represent that $\Psi_{\mathrm{H}}$ is the complex conjugate to $\Psi$, so we simply use $\Psi^{*}$ instead of $\Psi_{\mathrm{H}}$ in the case of imaginary $\mu$. Meanwhile, the first two equations indicate

$$
\frac{d \Omega^{*}}{d m_{0}}=\frac{d \Omega}{d m_{0}}, \quad \frac{d \Omega^{*}}{d \theta}=\frac{d \Omega}{d \theta},
$$

because $\sigma=d \Omega / d m_{0}$ and $n=-d \Omega / d \mu=i \beta d \Omega / d \theta$. The relations (19) are satisfied, only when $\Omega$ is real. Thus, $\Omega$ is real, so that $n$ is pure imaginary and $\sigma$ is real.

As shown in (16), $n$ is a function of $\cos (3 \theta)$ and $i \sin (3 \theta)$. This shows, together with the fact that $n$ is pure imaginary, that $n$ is $\theta$-odd (odd under the transformation $\theta \rightarrow-\theta$ ), since

$$
\begin{aligned}
& -n(\cos (3 \theta), i \sin (3 \theta))=n(\cos (3 \theta), i \sin (3 \theta))^{*} \\
& =n(\cos (3 \theta),-i \sin (3 \theta))=n(\cos (-3 \theta), i \sin (-3 \theta))
\end{aligned}
$$

Similarly, $\sigma$ is real and a function of $\cos (3 \theta)$ and $i \sin (3 \theta)$. This leads to the fact that $\sigma$ is $\theta$-even, because

$$
\begin{aligned}
& \sigma(\cos (3 \theta), i \sin (3 \theta))=\sigma(\cos (3 \theta), i \sin (3 \theta))^{*} \\
& =\sigma(\cos (3 \theta),-i \sin (3 \theta))=\sigma(\cos (-3 \theta), i \sin (-3 \theta)) .
\end{aligned}
$$

The potential $\Omega(\theta)$ depends on $\theta$ through $\Psi(\theta), \Psi(\theta)^{*}$, $\sigma(\theta), n(\theta)$ and $e^{3 i \theta}$. We then denote $\Omega(\theta)$ by $\Omega(\theta)=$ $\Omega\left(\Psi(\theta), \Psi(\theta)^{*}, n(\theta), e^{3 i \theta}\right)$, where $\sigma(\theta)$ is suppressed since it is $\theta$-even and does not make any influence on discussions shown below. Equation (14) keeps the same form under the transformation $\theta \rightarrow-\theta$, if $\Psi(\theta)$ and $\Psi(\theta)^{*}$ are replaced by $\Psi(-\theta)^{*}$ and $\Psi(-\theta)$, respectively. This indicates that

$$
\Psi(-\theta)=\Psi(\theta)^{*} \quad \text { and } \quad \Psi(-\theta)^{*}=\Psi(\theta) .
$$

Using these properties and the fact that $\Omega$ is real, one can show that

$$
\begin{aligned}
\Omega(\theta) & =(\Omega(\theta))^{*}=\Omega\left(\Psi(\theta)^{*}, \Psi(\theta),-n(\theta), e^{-3 i \theta}\right) \\
& =\Omega\left(\Psi(-\theta), \Psi(-\theta)^{*}, n(-\theta), e^{-3 i \theta}\right)=\Omega(-\theta) .
\end{aligned}
$$

Therefore, we can summarize that $\Omega$ is a periodic even function of $\theta$ with a period $2 \pi / 3, \Omega(\theta)=\Omega(\theta+2 \pi k / 3)=$ $\Omega(-\theta)$. The chiral condensate $\sigma(\theta)$ is also a periodic even function of $\theta$, while the quark number density $n$ is a periodic odd function of $\theta$. These properties are not changed by the presence/absence of the vector-type four-quark interaction, as understood from the proof shown above.

The real (imaginary) part of $\Psi$ is $\theta$-even ( $\theta$-odd), because

$$
\begin{aligned}
& \operatorname{Re}[\Psi(\theta)]=\left(\Psi(\theta)+\Psi(\theta)^{*}\right) / 2=\operatorname{Re}[\Psi(-\theta)], \\
& \operatorname{Im}[\Psi(\theta)]=\left(\Psi(\theta)-\Psi(\theta)^{*}\right) /(2 i)=-\operatorname{Im}[\Psi(-\theta)],
\end{aligned}
$$

where use has been made of (22). Thus, the real (imaginary) part of $\Psi$ is a periodic even (odd) function of $\theta$. Similarly, the absolute value $|\Psi|$ (phase $\phi$ ) of the Polyakov loop is a periodic even (odd) function of $\theta$, because $|\Psi|=$ $\sqrt{(\operatorname{Re}[\Psi])^{2}+(\operatorname{Im}[\Psi])^{2}}(\phi=\arctan (\operatorname{Im}[\Psi] / \operatorname{Re}[\Psi]))$. Obviously, these properties persist also in the case of $G_{\mathrm{v}}=0$.
Since all quantities of our interest have the RW periodicity, here we consider a period $0 \leq \theta \leq 2 \pi / 3$ in the $\theta-T$ plane. In the region, periodic even functions such as $\Omega(\theta)$, $\sigma(\theta), \operatorname{Re}[\Psi(\theta)]$ and $|\Psi|$ are symmetric with respect to a line $\theta=\pi / 3$. This indicates that such an even function $X_{\mathrm{e}}$ has a cusp at $\theta=\pi / 3$, if the gradient $d X_{\mathrm{e}} /\left.d \theta\right|_{\theta=\pi / 3 \pm \epsilon}$ is not zero, where $\epsilon$ is a positive infinitesimal. The phase transition appearing in $X_{\mathrm{e}}$ at $\theta=\pi / 3$ is of the second order. Meanwhile, $\operatorname{Im}[\Psi(\theta)], \phi$ and $n$ are periodic odd functions of $\theta$. This leads to the fact that these are discontinuous at $\theta=\pi / 3$, if the odd functions are not zero there. Thus, the phase transitions appearing in the odd functions at $\theta=\pi / 3$ are of the first order. These are seen in the high $T$ region, as shown later with numerical calculations. The phase transition appearing at $\theta=\pi / 3$ is called the RW transition. Thus, the RW phase transition is a family of first order transitions in $\theta$-odd quantities and second order ones in $\theta$-even quantities. This result is not changed by the presence/absence of vector-type four quark interaction.

As mentioned above, $\Omega$ of (14) is invariant under the extended $\mathbb{Z}_{3}$ transformation for any complex chemical potential $\mu=\mu_{\mathrm{R}}+i T \theta$. This implies that $X$ has the RW periodicity, $X\left(\mu_{\mathrm{R}}+i T \theta\right)=X\left(\mu_{\mathrm{R}}+i T(\theta+2 \pi k / 3)\right)$. Actually, $\Omega$ depends on $\theta$ only through the factor $\exp (3 i \theta)$, even if $\mu_{\mathrm{R}}$ is nonzero. Hence, the solutions $X$ of $\partial \Omega / \partial X=0$ are functions of $\exp (3 i \theta)$. Thus, the concept of the extended $\mathbb{Z}_{3}$ transformation is useful for any complex chemical potential.

Finally, we briefly consider the case of real $\mu$ by taking $\theta=0$ in (14); here note that $\Psi=\Phi$ and $\Psi_{\mathrm{H}}=\Phi_{\mathrm{H}}$. The complex conjugate potential $\Omega^{*}$ has the same form as $\Omega$, if $\sigma^{*}$, $n^{*}, \Psi^{*}$ and $\Psi_{\mathrm{H}}^{*}$ are replaced by $\sigma, n, \Psi$ and $\Psi_{\mathrm{H}}$, respectively. This indicates that the solutions $X^{*}$ of $\partial \Omega^{*} / \partial X^{*}=0$ are related to the solutions $X$ of $\partial \Omega / \partial X=0$ as

$$
\sigma^{*}=\sigma, n^{*}=n, \quad \Psi^{*}=\Psi, \quad \Psi_{\mathrm{H}}^{*}=\Psi_{\mathrm{H}} .
$$

Thus, the four quantities are real. Furthermore, the first and second equations of 25) are reduced to

$$
\frac{d \Omega^{*}}{d m_{0}}=\frac{d \Omega}{d m_{0}}, \quad \frac{d \Omega^{*}}{d \mu_{\mathrm{R}}}=\frac{d \Omega}{d \mu_{\mathrm{R}}}
$$

respectively. This indicates that $\Omega$ is also real.

Equation (14) keeps the same form under the transformation $\mu_{\mathrm{R}} \rightarrow-\mu_{\mathrm{R}}$, if $\Psi, \Psi_{\mathrm{H}}, \sigma$ and $n$ are replaced by $\Psi_{\mathrm{H}}$, $\Psi, \sigma$ and $-n$, respectively. This indicates that the solutions $X\left(-\mu_{\mathrm{R}}\right)$ of $\partial \Omega\left(-\mu_{\mathrm{R}}\right) / \partial X=0$ are related to the solutions $X\left(\mu_{\mathrm{R}}\right)$ of $\partial \Omega\left(\mu_{\mathrm{R}}\right) / \partial X=0$ as

$$
\begin{aligned}
\Psi_{\mathrm{H}}\left(-\mu_{\mathrm{R}}\right) & =\Psi\left(\mu_{\mathrm{R}}\right), \quad \Psi\left(-\mu_{\mathrm{R}}\right)=\Psi_{\mathrm{H}}\left(\mu_{\mathrm{R}}\right), \\
\sigma\left(-\mu_{\mathrm{R}}\right) & =\sigma\left(\mu_{\mathrm{R}}\right), \quad n\left(-\mu_{\mathrm{R}}\right)=-n\left(\mu_{\mathrm{R}}\right) .
\end{aligned}
$$

Using (27) in (14), we can easily confirm that $\Omega\left(\mu_{\mathrm{R}}\right)=$ $\Omega\left(-\mu_{\mathrm{R}}\right)$. Thus, $\Omega$ and $\sigma$ are even functions of $\mu$ in both the real and imaginary $\mu$ regions, so the chiral transition curve shown later is plotted in the $\mu^{2}-T$ plane. 


\section{NUMERICAL RESULTS}

We proceed to numerical calculations. Since the PNJL model are nonrenormalizable, it is then needed to introduce a cutoff in the momentum integration. Here we take a threedimensional momentum cutoff $\Lambda$. Hence, the present model has four parameters $m_{0}, \Lambda, G_{\mathrm{S}}, G_{\mathrm{v}}$ in the NJL sector. Following Ref. [29], we take $m_{0}=5.5 \mathrm{MeV}, \Lambda=0.6315 \mathrm{GeV}$ and $G_{\mathrm{S}}=5.498 \mathrm{GeV}^{-2}$ that reproduce the pion decay constant $f_{\pi}=93.3 \mathrm{MeV}$ and the pion mass $M_{\pi}=138 \mathrm{MeV}$. That is, we pin down parameters other than $G_{\mathrm{v}}$ at $\mu=0$, then we treat $G_{\mathrm{v}}$ as a free parameter and vary its value in the range $0 \leq G_{\mathrm{v}} \leq G_{\mathrm{s}}$.

Figure 1 represents the RW periodicity of various quantities calculated with/without the vector-type interaction. These graphs clearly shows that the RW periodicity is not affected by the inclusion of the vector-type interaction as argued analytically. At $T=170 \mathrm{MeV}$, which is below the endpoint of the RW phase transition (point A of Fig. 3), all quantities are smooth as functions of $\theta$, whereas at $T=250 \mathrm{MeV}$, which is above the endpoint, the $\theta$-even quantities, $\sigma$ and $\operatorname{Re}[\Psi]$, exhibit second-order and the $\theta$-odd quantities, $\operatorname{Im}[n]$ and $\operatorname{Im}[\Psi]$, do first-order phase transitions. We note here that the present endpoint $\mathrm{A}$ is lower than in our previous works [6] because $T_{0}$ in the Polyakov potential was rescaled in the present calculation.

Next we pay attention to the impact of the vector-type interaction on various quantities. Figure 2 represents the four quantities same as in Fig. 1 for half a RW period, $0 \leq \theta \leq$ $\pi / 3$, calculated with various strengths $G_{\mathrm{v}}$. Note that here we present the results at $T=300 \mathrm{MeV}$ since the effect of the vector-type interaction is more conspicuous at higher temperatures. First of all, the chiral condensate shown in Fig. 2(a), in particular around the RW transition at $\theta=\pi / 3$, is sensitive to $G_{\mathrm{v}}$. The calculated result means that the vector-type interaction reduces the constituent quark mass $M$. The vectortype interaction suppresses the effective chemical potential $\tilde{\mu}=i \theta T-2 i G_{\mathrm{v}} \operatorname{Im}[n]$. This directly affects $\operatorname{Im}[n]$, namely, $\operatorname{Im}[n]$ decreases as $G_{\mathrm{v}}$ increases. Although the indirect effect through $M$, mentioned just above, works oppositely, the direct effect is stronger and therefore survives. Consequently effects of the vector-type interaction on $\operatorname{Im}[n]$ shown in Fig. 2(b) as well as on $\sigma$ are significant. Figures 2 (c) and (d) indicate that the effects on the modified Polyakov loop are negligible because it is fully determined by the Polyakov potential. The physical value of $G_{\mathrm{v}}$ is still an open matter although its effect is significant in the physical real- $\mu$ phase diagram (see Fig. 4 below). Since the present calculation shows that its impacts on the chiral condensate and the quark number density are appreciable in the imaginary- $\mu$ region where both lattice simulations and effective model calculations are available, we expect that it would be possible to determine a physical strength of $G_{\mathrm{v}}$ by comparing both the results, although the former is not available yet for the case of the two flavor QCD.

We report the impact of the vector-type four-quark interaction on the $\mu^{2}-T$ phase diagram ranging from negative to positive $\mu^{2}$ in Fig. 3, since the order parameters $\sigma$ are functions of $\mu^{2}$ as proved analytically above. This figure indi-
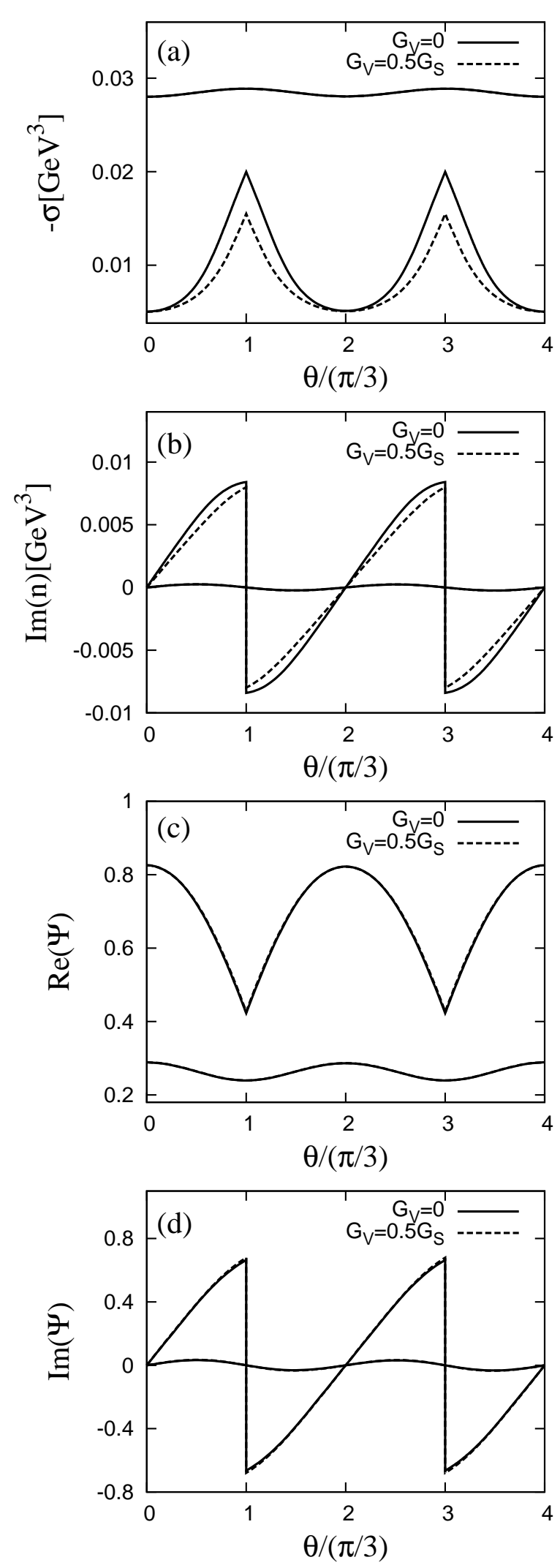

Fig. 1: The Roberge-Weiss periodicity of (a) chiral condensate, (b) imaginary part of the quark number density, (c) real part of the modified Polyakov loop, and (d) its imaginary part. The solid curves are for $G_{\mathrm{v}}=0$ and the dashed ones are for $G_{\mathrm{v}}=0.5 G_{\mathrm{s}}$. The curves with cusps ( $\theta$-even quantities) and those with discontinuities ( $\theta$-odd quantities) are for $T=250 \mathrm{MeV}$, while smooth ones are for $T=170 \mathrm{MeV}$. 

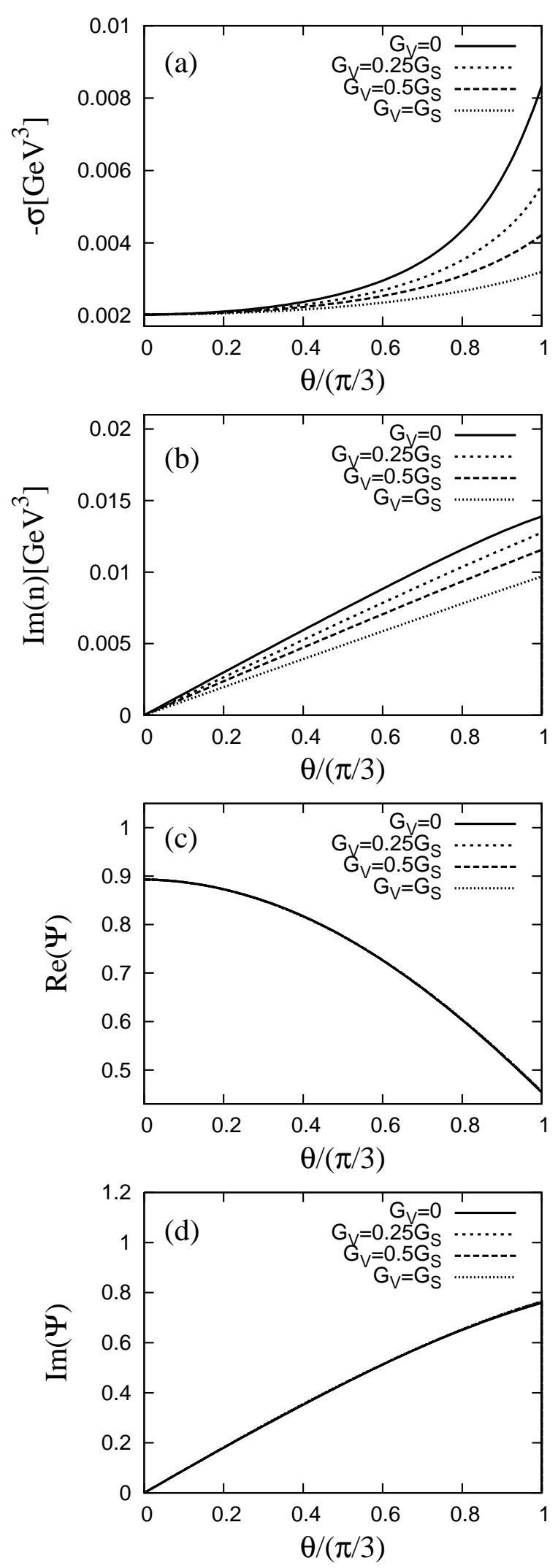

Fig. 2: The impact of the vector-type four-quark interaction on the four quantities same as in Fig. 1 at $T=300 \mathrm{MeV}$ for a half RobergeWeiss period. cates that effect of the vector-type interaction on the phase diagram is visible in the real- $\mu$ part; it has been known that the critical endpoint (CEP; point C) moves to lower $T$ and higher $\mu$ as $G_{\mathrm{V}}$ increases and eventually disappears at $G_{\mathrm{V}} \approx$ $0.38 G_{\mathrm{S}}[22,24,28,29]$. In contrast, its effects are less in the imaginary- $\mu$ part of the phase diagram although the values of $\sigma$ and $\operatorname{Im}[n]$ are sensitive to it. This can be understood from the fact that the effect of finite $G_{\mathrm{v}}$ on $\tilde{\mu}$ is important when $T$ is low whereas the RW transition occurs at higher $T$. We note here that this figure graphs the chiral transition curve although the deconfinement transition temperature in the imaginary- $\mu$ is lower. We confirmed that their difference decreases as $\theta$ increases due to finite $G_{\mathrm{v}}$.
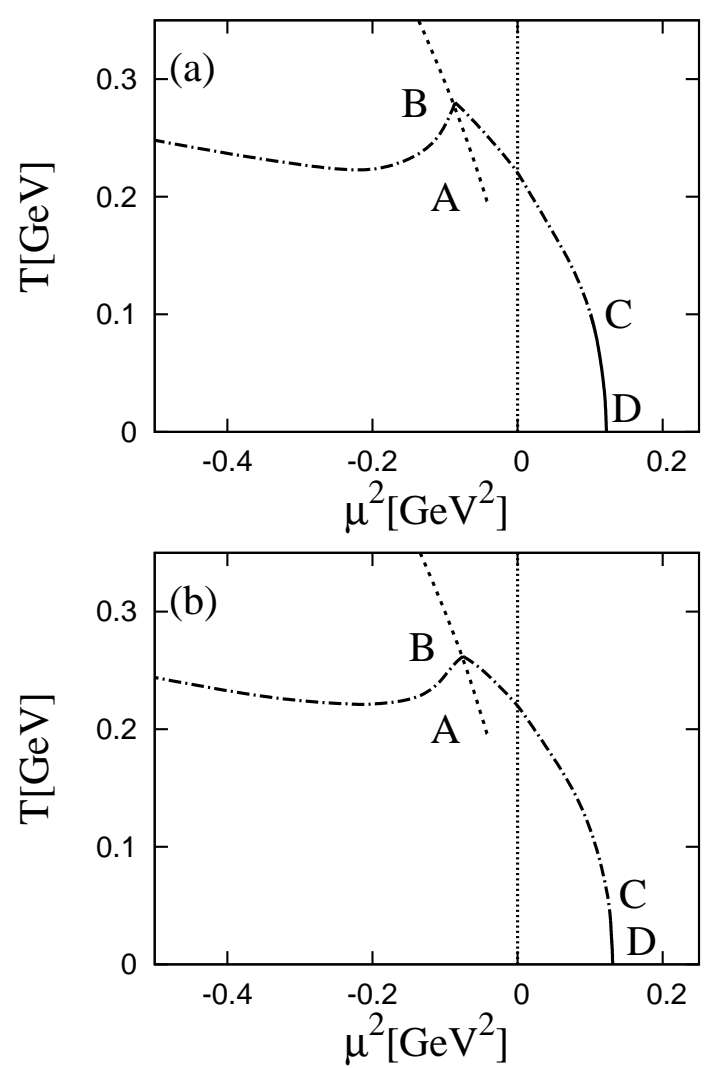

Fig. 3: The phase diagram in the $\mu^{2}-T$ plane, (a) for $G_{\mathrm{v}}=0$ and (b) for $G_{\mathrm{V}}=0.25 G_{\mathrm{s}}$. The point A is the endpoint of the RW phase transition, $\mathrm{B}$ is the crossing point of the ordinary chiral crossover transition curve and the RW phase transition line, $\mathrm{C}$ is the chiral critical endpoint, and $\mathrm{D}$ is the chiral transition point at $T=0$.

Finally, in order to look into the correlation between the key quantities in the imaginary- and real- $\mu$ regions, we present in Fig. 4 the relation between the chiral condensate at $\theta=$ $\pi / 3-\epsilon$ and $T=250 \mathrm{MeV}$ in the imaginary- $\mu$ region, which can be directly measured in lattice simulations, and the position of the chiral CEP in the real- $\mu$ region, which cannot be measured. Both of them are functions of $G_{\mathrm{V}}$; here $\epsilon$ is a positive infinitesimal. The larger $G_{\mathrm{v}}$ is adopted, the smaller $-\sigma$ becomes. The right-most point corresponds to $G_{\mathrm{v}}=0$ while the left-most does to $G_{\mathrm{v}}=0.30 G_{\mathrm{s}}$. Since the correlation 
shown in Fig. 4 is evident, we hope this serves to determine the physical value of $G_{\mathrm{v}}$.

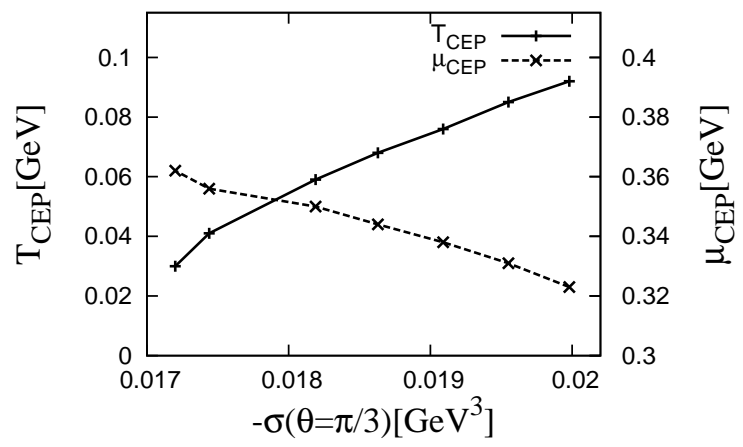

Fig. 4: The correlation between the chiral condensate at $\theta=\pi / 3-\epsilon$ in the imaginary- $\mu$ region and the position of the chiral critical endpoint (CEP) in the real- $\mu$ region, where $\epsilon$ is a positive infinitesimal. These curves correspond to $0 \leq G_{\mathrm{v}} \leq 0.30 G_{\mathrm{s}}$.

\section{SUMMARY}

A key feature of QCD in the imaginary chemical potential $(\mu)$ region is that the partition function has the extended $\mathbb{Z}_{3}$ symmetry. We prove that the Polyakov-loop extended
Nambu-Jona-Lasinio (PNJL) model possesses the symmetry, even if the vector-type four-quark interaction is newly added to the NJL sector of the PNJL Lagrangian. Thus, PNJL is a suitable model to analyze not only the real $\mu$ region but also the imaginary one.

Using PNJL, we have analyzed the effect of the vector-type four-quark interaction in the imaginary $\mu$ region and investigated the correlation between the position of the critical endpoint (CEP) in the real $\mu$ region and several quantities in the imaginary $\mu$ region. In the real $\mu$ region, the position of CEP is known to be sensitive to the strength of the vector-type fourquark interaction. Meanwhile, the present analysis shows that in the imaginary $\mu$ region the vector-type four-quark interaction largely changes values of the chiral condensate and the quark-number density. This indicates that these quantities in the imaginary $\mu$ region are suitable to determine the strength and therefore the QCD phase diagram in the real $\mu$ region.

\section{Acknowledgments}

The authors thank A. Nakamura for useful discussions and suggestions. K.K. and H.K. also thank M. Imachi, H. Yoneyama and M. Tachibana for useful discussions. This work has been supported in part by the Grants-in-Aid for Scientific Research (18540280) of Education, Science, Sports, and Culture of Japan.
[1] J. Kogut, M. Stone, H. W. Wyld, W. R. Gibbs, J. Shigemitsu, S. H. Shenker, and D. K. Sinclair, Phys. Rev. Lett. 50, 393 (1983).

[2] J. B. Kogut and D. K. Sinclair Phys. Rev. D 77, 114503 (2008).

[3] P. de Forcrand and O. Philipsen, Nucl. Phys. B642, 290 (2002); P. de Forcrand and O. Philipsen, Nucl. Phys. B673, 170 (2003).

[4] M. D'Elia and M. P. Lombardo, Phys. Rev. D 67, 014505 (2003); M. D'Elia and M. P. Lombardo, Phys. Rev. D 70, 074509 (2004); M. D'Elia, F. D. Renzo, and M. P. Lombardo, Phys. Rev. D 76, 114509 (2007); M. P. Lombardo, arXiv:heplat/0612017 (2006).

[5] H. S. Chen and X. Q. Luo, Phys. Rev. D72, 034504 (2005); L. K. Wu, X. Q. Luo, and H. S. Chen, Phys. Rev. D76, 034505 (2007).

[6] Y. Sakai, K. Kashiwa, H. Kouno, and M. Yahiro, Phys. Rev. D 77, 051901 (2008); arXiv:hep-ph/0803.1902 (2008), to be published in Phys. Rev. D.

[7] A. Roberge and N. Weiss, Nucl. Phys. B275, 734 (1986).

[8] Y. Nambu and G. Jona-Lasinio, Phys. Rev. 122, 345 (1961); Phys. Rev. 124, 246 (1961).

[9] P. N. Meisinger, and M. C. Ogilvie, Phys. Lett. B 379, 163 (1996).

[10] A. Dumitru, and R. D. Pisarski, Phys. Rev. D 66, 096003 (2002); A. Dumitru, Y. Hatta, J. Lenaghan, K. Orginos, and R. D. Pisarski, Phys. Rev. D 70, 034511 (2004); A. Dumitru, R. D. Pisarski, and D. Zschiesche, Phys. Rev. D 72, 065008 (2005).

[11] K. Fukushima, Phys. Lett. B 591, 277 (2004).

[12] S. K. Ghosh, T. K. Mukherjee, M. G. Mustafa, and R. Ray, Phys. Rev. D 73, 114007 (2006).
[13] E. Megías, E. R. Arriola, and L. L. Salcedo, Phys. Rev. D 74, 065005 (2006).

[14] C. Ratti, M. A. Thaler, and W. Weise, Phys. Rev. D 73, 014019 (2006).

[15] M. Ciminale, R. Gatto, N. D. Ippolito, G. Nardulli, and M. Ruggieri, Phys. Rev. D 77, 054023 (2008); M. Ciminale, G. Nardulli, M. Ruggieri, and R. Gatto, Phys. Lett. B 657, 64 (2007).

[16] C. Ratti, S. Rößner, M. A. Thaler, and W. Weise, Eur. Phys. J. C 49, 213 (2007).

[17] S. Rößner, C. Ratti, and W. Weise, Phys. Rev. D 75, 034007 (2007).

[18] H. Hansen, W. M. Alberico, A. Beraudo, A. Molinari, M. Nardi, and C. Ratti, Phys. Rev. D 75, 065004 (2007).

[19] C. Sasaki, B. Friman, and K. Redlich, Phys. Rev. D 75, 074013 (2007).

[20] B. J. Schaefer, J. M. Pawlowski, and J. Wambach, Phys. Rev. D 76, 074023 (2007).

[21] W. J. Fu, Z. Zhang, and Y. X. Liu, Phys. Rev. D 77, 014006 (2008).

[22] K. Kashiwa, H. Kouno, M. Matsuzaki, and M. Yahiro, Phys. Lett. B 662, 26 (2008).

[23] P. Costa, C. A. de Sousa, M. C. Ruivo, and H. Hansen, arXiv:hep-ph/0801.3616 (2008).

[24] K. Fukushima, arXiv:hep-ph/0803.3318 (2008).

[25] K. Kashiwa, Y. Sakai, H. Kouno, M. Matsuzaki, and M. Yahiro, arXiv:hep-ph/0804.3557 (2008)

[26] H. Abuki, M. Ciminale, R. Gatto, N. D. Ippolito, G. Nardulli, and M. Ruggieri, arXiv:hep-ph/0801.4254 (2008); H. Abuki, M. Ciminale, R. Gatto, G. Nardulli, and 
M. Ruggieri, arXiv:hep-ph/0802.2396 (2008); H. Abuki, R. Anglani, R. Gatto, G. Nardulli, and M. Ruggieri, arXiv:hepph/0805.1509 (2008); H. Abuki, arXiv:hep-ph/0805.3076 (2008).

[27] M. Asakawa and K. Yazaki, Nucl. Phys. A504, 668 (1989).

[28] M. Kitazawa, T. Koide, T. Kunihiro, and Y. Nemoto, Prog. Theor. Phys. 108, 929 (2002).

[29] K. Kashiwa, H. Kouno, T. Sakaguchi, M. Matsuzaki, and M. Yahiro, Phys. Lett. B 647, 446 (2007); K. Kashiwa, M. Matsuzaki, H. Kouno, and M. Yahiro, Phys. Lett. B 657, 143 (2007).

[30] J. D. Walecka, Ann. Phys. 83, 491 (1974).

[31] T. Kashiwa and T. Sakaguchi, Phys. Rev. D 68, 065002 (2003).

[32] T. Sakaguchi, M. Matsuzaki, H. Kouno, and M. Yahiro, Centr.
Eur. J. Phys. 6, 116 (2008).

[33] H. Kouno, T. Sakaguchi, K. Kashiwa, M. Hamada, H. Tokudome, M. Matsuzaki, and M. Yahiro, Soryushiron Kenkyu 112 C67 (arXiv:nucl-th/0509057) (2005).

[34] G. Boyd, J. Engels, F. Karsch, E. Laermann, C. Legeland, M. Lütgemeier, and B. Petersson, Nucl. Phys. B469, 419 (1996).

[35] O. Kaczmarek, F. Karsch, P. Petreczky, and F. Zantow, Phys. Lett. B 543, 41 (2002).

[36] F. Karsch, Lect. notes Phys. 583, 209 (2002).

[37] F. Karsch, E. Laermann, and A. Peikert, Nucl. Phys. B 605, 579 (2002).

[38] M. Cheng et al., Phys. Rev. D 74, 054507 (2006). 